\title{
ZONIFICACIÓN AMBIENTAL DE LA RESERVA NATURAL BAHÍA SAN ANTONIO, ARGENTINA. APLICACIÓN DEL ÍNDICE DE CALIDAD AMBIENTAL
}

\author{
María E. Carbone ${ }^{1,2}$, María C. Piccolo ${ }^{1,2}$, Gerardo M.E. Perillo ${ }^{1,3}$ \\ ${ }^{1}$ Instituto Argentino de Oceanografía, Bahía Blanca, Argentina \\ ${ }^{2}$ Departamento de Geografía y Turismo UNS \\ ${ }^{3}$ Departamento de Geología UNS Bahía Blanca, Argentina
}

\section{RESUMEN}

Las actividades humanas y los procesos naturales son los elementos necesarios en los cambios que afectan a los diferentes ambientes en las zonas costeras patagónicas. El objetivo de este trabajo es determinar la calidad ambiental actual de las localidades que conforman la reserva bahía San Antonio. Mediante la aplicación del Índice de Calidad Ambiental (EQI). Los resultados obtenidos permitieron zonificar el área de la siguiente manera: San Antonio Oeste donde el índice oscila entre un valor de 0.50 y 0.45 , refleja una mayor actividad humana relacionada a la extracción de recursos mineros, pesqueros. La zona de San Antonio Este posee un índice de 0.34 como resultado de las actividades portuarias y características de sitio. En tercer lugar Las Grutas posee valores de hasta 0.28 que muestra una gran actividad turística anual, una escasa planificación urbana y sectores con problemas de erosión marcados principalmente en los acantilados. La cuarta zona determinada corresponde al sector norte de bahía San Antonio con valores de hasta 0.63 donde las actividades humanas son escasas y la circulación del flujo de agua favorece la renovación constante del ambiente. Se evidencia una importante alteración de la condición natural de estos ecosistemas generada por la sobreexplotación del medio físico, contaminación del mismo y la escasa aplicación de políticas ambientales y urbanas en el pasado cercano.

Palabras clave: indicadores, calidad ambiental, pautas de manejo, Bahía San Antonio.

\section{ABSTRACT}

Human activities and natural processes are needed in changes affect different environments in coastal Patagonia. The aim of this study is to determine the current environmental quality places that make San Antonio Bay reserve. Applying the Environmental Quality Index (EQI). Results obtained on study areas as follows: San Antonio West where the index ranges from a value of 0.50 and 0.45 , reflecting increased human activity related to the extraction of mineral resources, fisheries. The area of San 
Antonio East has an index of 0.34 as a result of port activities and site features. Third class has values up to 0.28 which shows a large annual tourist activity, poor urban planning and erosion problem in the cliffs. The fourth specific area corresponding to the northern sector of San Antonio Bay with values up to 0.63 which human activities are scarce and the water flow circulation the constant of the environment. It shows a significant alteration of the natural condition of these ecosystems caused by overfishing of the physical environment and the contamination of environmental and urban policies in the recent past. Bay.

Key words: indicators, environmental quality, management guidelines, San Antonio

\section{Introducción}

Las actividades humanas y los procesos naturales son los elementos necesarios en los cambios que afectan a los diferentes ambientes en las zonas costeras. Las consecuencias de esos cambios se aprecian en la calidad ambiental intrínseca de esas áreas, debido a la gran diversidad de unidades ecológicas asociadas a la productividad biológica. Los procesos naturales que intervienen hoy en la evolución de las zonas costera están asociados principalmente a la erosión, sedimentación y a la variación del nivel del mar. Actualmente los diferentes procesos humanos son el principal agente de las alteraciones, la presión se ejerce a través de la ocupación territorial costera, ya sea en grandes dimensiones o en escalas menores como ocurre con balnearios estivales (Rivas y Cendrero, 1991 y 1995, Charlier y Bologa, 2003 y Rivas et al. 2006).

Las transformaciones producen un deterioro mayor de esos ambientes cuando no están acompañados de un adecuado sistema de pautas de manejo ordenado y sustentable. La formulación e implementación de medidas adecuadas de planeamiento y manejo costero surge de un diagnóstico actual concreto que pueda proyectar futuros cambios y modificaciones en este tipo de ambientes. El área costera puede abordarse desde tres perspectivas desagregadas: componentes ambientales, características morfodinámicas actuantes y actuales y por último el nivel juridiccional y administrativo correspondiente al área de estudio (Rivas et al. 2006; Maelfait, et al., 2006).

Los cambios en el uso del suelo, la ampliación de equipamientos e infraestructura de los asentamientos humanos ocasionan cambios rápidos en las zonas costeras. La interrelación entre las características naturales y las diversas actividades humanas en estas zonas genera conflictos entre la conservación y el desarrollo. La necesidad de compatibilizar sendos conceptos es muy importante en aquellas zonas con elevada presión de ocupación territorial y presencia de recursos naturales vulnerables (Cendrero y Fischer, 1997, Berger y Liverman, 2002, Bonachea et al, 2003) como sucede en las zonas costeras patagónicas argentinas.

El planteo e implementación de programas y políticas para un manejo costero sustentable de estas zonas se ve fortalecido con un adecuado monitoreo de la calidad ambiental del sitio. Para ello se algunos autores plantean aplicar indicadores de tipo cuantitativo que reflejan las características o cualidades significativas de los diferentes ambientes, que se integran en índices numéricos agregados que permitan establecer una herramienta útil y precisa para poder adoptar estrategias de planificación y manejo costero. (OECD, 1994, UNDP, 1995, SCOPE,1995; Cendrero y Fisher, 1997, Jiménez Beltrán, 2000, Pirrone et al. 2005, Maelfait, et al., 2006, Orpi et al. 2006).

Algunos autores integran y cuantifican los indicadores para obtener el grado de demanda ejercido sobre el medio ribereño o costero a partir del incremento demográfico, de cambios de usos del suelo, incremento en el consumo del agua principalmente en aquellos ambientes 
caracterizados por un déficit hídrico marcado (Laredo et al., 2001, Bellot et al., 2006, Chirino et al, 2008). La aplicación de estos índices también permite analizar los cambios ambientales que sufren los diferentes humedales durante periodos extensos comparables (Lin et al.,2007) donde se determinan que tipo de actividades e impactos antropicos los produjeron.

En Argentina estos estudios integrados en ambientes costeros no son muy numerosos, se pueden mencionar a Cendrero et al (2003), Rivas et al (2006) Becker, (2006) que aplicaron indicadores e índices interrelacionados comparando ambientes costeros similares de este país, con México y España. Además proponen implementar pautas de manejo costero ya aplicados en dichos países, en una zona de playas del sudeste de la provincia de Buenos Aires. Otros autores aplican este índice a partir de problemáticas naturales basada en procesos de erosión y vulnerabilidad de costas arenosas asociados a cambios relativos del nivel del mar (Marcomini et al., 2007, Iantanos N, 2004, Dadon, 2006 y Dadón y Matteuci, 2006, 2007). En este trabajo de investigación se pretende determinar la calidad ambiental actual de las localidades que integran un área protegida costera norpatagónica: la reserva natural bahía San Antonio y área de adyacencia, a través del índice de calidad Ambiental (EQI). Este índice surge de la interrelación entre los índices que abarcan variables naturales (IN) y los de las variables derivadas de las fuentes de recursos (IR), tratamiento de residuos (IW) y de las actividades y servicios (ISS) que se interrelacionan en este ambiente costero.

\section{1. Área de estudio}

La Bahía San Antonio ubicada en el extremo noroeste del Golfo San Matías, provincia de Río Negro, fue declarada Reserva Natural en junio de 1993 y Reserva Internacional de la Red Hemisférica de Reservas para Aves Playeras (RHRAP). Incluye tres localidades San Antonio Oeste, San Antonio Este y el Balneario Las Grutas, además del área de adyacencia. Su morfología está integrada por planicies y canales de mareas, cordones, espigas, playas y médanos (Figura 1). Al norte, la bahía está bordeada por una planicie baja, conformada por espigas de rodados y arenas que se entremezclan con las planicies de marea. Son en su mayor parte, llanuras de fango intermareal sobre las que se observa una densa red de canales de marea. En las cabeceras de estos últimos se evidencian procesos que contribuyen a la sedimentación de los mismos. Al sur, las costas desarrolladas en las espigas mencionadas se destacan por los fenómenos de acreción de sus costas (Carbone et al., 2007, 2008). En ambas márgenes de la bahía se emplazan dos puertos: el de San Antonio Oeste con construcción precaria para el amarre de buques pesqueros de poco calado y eslora y el de San Antonio Este que es un puerto de ultramar de aguas profundas con intensa actividad exportadora de fruta (pera, manzana, ciruela, durazno, pelón, uva, limón), jugo, cebollas, ajo y pescado.

En el área cercana al Balneario Las Grutas los acantilados no superan los $10 \mathrm{~m}$ de altura, tienen rasgos erosivos en retracción como consecuencia de la acción hídrica continental evidenciada por la presencia de cañadones. La red de drenaje en este sector posee cauces cortos aproximadamente perpendiculares a la costa producto de un régimen fluvial temporal debido a las escasas precipitaciones regionales (Genchi et al., 2008). Hacia el sur del balneario se observa la presencia de acantilados activos con muescas en la base. Este tipo de erosión es generada por la acción de las olas y marea junto a la abrasión física de los rodados existentes en el sector de playas. A lo largo de todo este sector mencionado además de los acantilados y depósitos arenosos, aparecen en forma continua plataformas de abrasión rocosas que, en algunos sectores, se presentan parcialmente cubiertas por materiales sueltos tales como arenas, gravas, etc. 


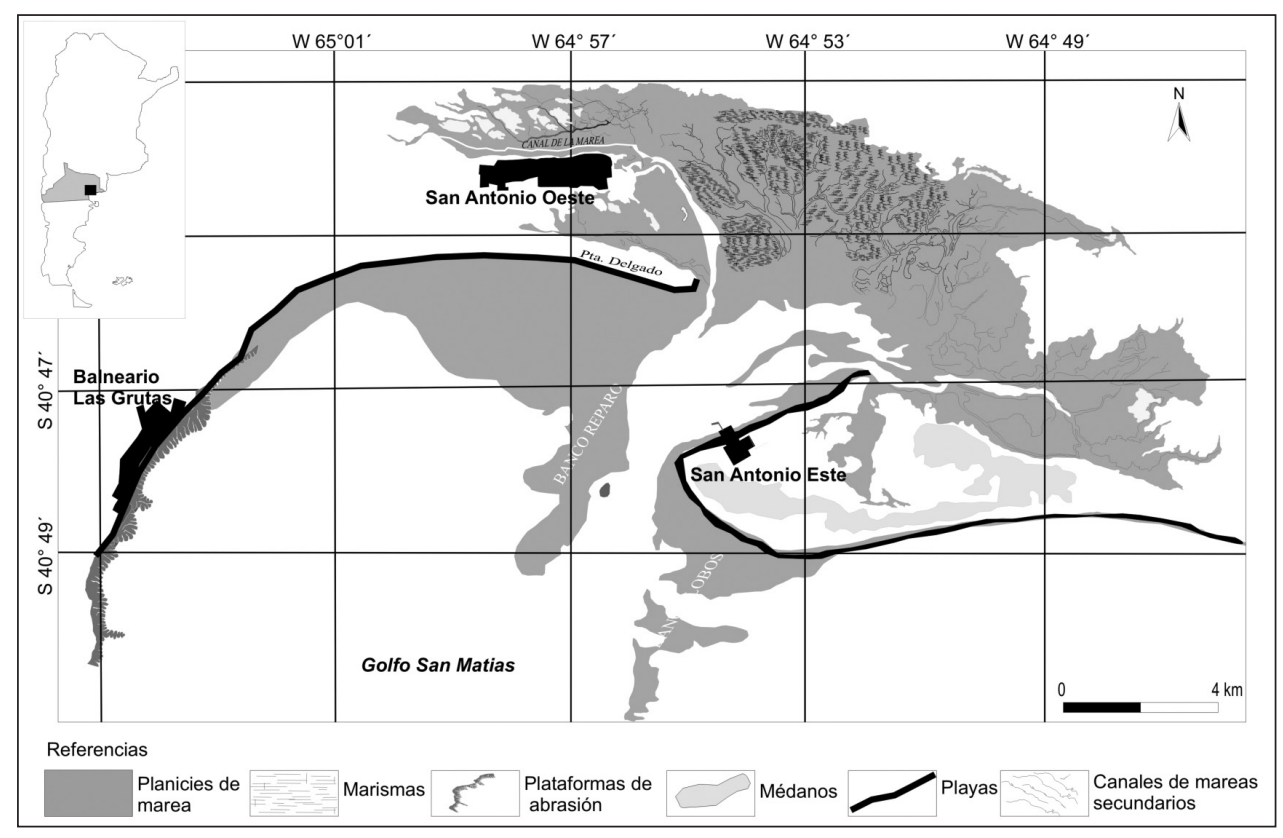

FiguRa 1. Unidades geomorfológicas de la reserva natural Bahía San Antonio.

El clima es semiárido con distribución irregular de precipitaciones. La temperatura media anual es de $13^{\circ}$ (SNM, 1991). Predominan los suelos moderadamente someros, de textura franco-arenosa, arenosa y arcillosa, comúnmente con carbonato de calcio, muy escasa de materia orgánica, de $\mathrm{pH}$ moderadamente alcalinos y de un fuerte déficit hídrico anual. En las áreas relativamente bajas de las planicies aluviales dominan suelos salinos-alcalinos. Es frecuente la presencia de procesos de erosión combinada eólica-hídrica moderada (Inta, 1999). La vegetación corresponde a la provincia fitogeográfica del Monte Austral. La fisonomía predominante es la de una estepa arbustiva media (arbustos de 1 a $2 \mathrm{~m}$ de altura), con una cobertura vegetal total del 20 al 40\%. Los principales componentes florísticos son: la jarilla (Larrea divaricata), la jarilla macho (Larrea cuneifolia), la zampa (Atriplex lampa), el alpataco (Prosopis alpataco), el molle (Schinus polygamus) y el monte negro o uña de gato (Bougainvillea spinosa). Por debajo de estos arbustos las especies más comunes son el tomillo (Acantholippa seriphioides), el olivillo (Hyalis argentea), el coirón amargo, el coirón pluma y la flechilla (Stipa tenuis). Las principales actividades económicas son la ganadería ovina y caprina y en algunos sectores bovina. En las zonas costeras predominan la pesca y el turismo.

\section{Materiales y métodos}

El procedimiento metodológico empleado para determinar el EQI en la reserva natural bahía San Antonio consta de tres etapas fundamentales. En la primera etapa se seleccionaron y elaboraron 53 indicadores (Tabla 1) para los años 2007 y 2008, obtenidos a partir de: a. cartas topográficas escala 1:100.000, cartas de imagen satelitaria 1:250.000, fotografías aéreas escala 1:20.000, imágenes satelitales Landsat 5 y 7 procesadas, b. mediciones y ob- 
servación directa en terreno (a través de campañas de relevamiento realizadas a la zona de estudio), c. antecedentes bibliográficos específicos y d. información estadística a partir de Censo de Hogar y Vivienda 2001, Censo Agropecuario 2004, Estadísticas Meteorológicas 1991-2000, Información de la Municipalidad de San Antonio Oeste (SAO) y las delegaciones municipales de San Antonio Este (SAE) y del balneario Las Grutas (LG).

Se aplica el modelo DPSIR (EEA, 1998, SCOPE, 1995) que da un marco de organización de la información para estructurar los indicadores. Implica elaborar de manera general una progresión causal de las acciones humanas que ocasionan una presión sobre el medio ambiente y los recursos naturales, que llevan a un cambio en el estado del medio ambiente y al que la sociedad responde con medidas o acciones para reducir o prevenir el impacto. Se entiende como grado de naturalidad (IN) de una región, la ausencia de modificaciones introducidas por el hombre, se puede concebir el papel del medio físico como fuente de diversos recursos (IR), como sumidero de distintos tipos de residuos (IW) generados por las actividades humanas (ISS) y como soporte de dichas actividades (OEDC, 1994).

Durante la segunda etapa se normalizaron los datos de los indicadores ambientales seleccionados, se asignó una escala nominal de 0 a 1 , donde 0 corresponde a la peor situación posible con respecto al indicador en cuestión y 1 a la mejor situación posible con respecto al indicador analizado según la siguiente expresión:

$$
\mathrm{V}=(\mathrm{Im}-\operatorname{Imin}) /(\operatorname{Imax}-\mathrm{Imin})
$$

Donde V: valor normalizado, Im: valor del indicador, Imax: valor máximo en la zona de estudio; Imin: valor mínimo en la zona

Tabla 1

LISTADO DE INDICADORES POR UNIDAD ADMINISTRATIVA

(MODIFICADO DE OECD, 1995, CENDRERO ET AL 2003)

\begin{tabular}{|c|c|c|c|}
\hline Variables & indicadores & unidades & $\begin{array}{c}\text { fuente del } \\
\text { dato }\end{array}$ \\
\hline $\begin{array}{c}\text { naturalidad } \\
\text { presión } \\
\text { estado }\end{array}$ & $\begin{array}{l}\text { 1. densidad de rutas y caminos } \\
\text { 2. geoformas/ecosistemas naturales } \\
\text { 3. n de especies vertebrados de interés } \\
\text { 4. contaminación del agua (canals de } \\
\text { marea) } \\
\text { 5. modificaciones de geoambientes } \\
\text { 6. riesgo de contaminación acuíferos } \\
\text { 7. área protegida }\end{array}$ & $\begin{array}{l}\mathrm{km} / \mathrm{km}^{2} \\
\mathrm{n}^{\circ} \text { de polígonos } / \mathrm{km}^{2} \\
\% \\
\% \text { longitud del rio/canales } \\
\%, \text { valores de erosion } \\
\%, \mathrm{~m}^{2} / \text { persona } \\
\%, \mathrm{~m}^{2} / \text { persona }\end{array}$ & $\begin{array}{l}a \\
b \\
c \\
a-c \\
a-b \\
b-c \\
a-c-d\end{array}$ \\
\hline $\begin{array}{c}\text { fuentes de recursos } \\
\text { presión }\end{array}$ & $\begin{array}{l}\text { 8. áreas cultivadas } \\
\text { 9. extracción de especies claves fauna y } \\
\text { flora } \\
\text { 10. área de desoves } \\
\text { 11. área de minería } \\
\text { 12. densidad de población con respecto a } \\
\text { suelos cultivables } \\
\text { 13. campos de suelos aptos } \\
\text { 14. caudales dulces (canal Pomona) }\end{array}$ & $\begin{array}{l}\% \\
\mathrm{~kg} / \mathrm{km}^{2} \\
\% \\
\% \\
\mathrm{n}^{\circ} / \mathrm{km}^{2} \\
\mathrm{n}^{\circ} / \mathrm{km}^{2} \\
\mathrm{~m}^{3} / \mathrm{año}\end{array}$ & $\begin{array}{l}a-c-d \\
c \\
c \\
a-c \\
a-c \\
a-c \\
a-c-d\end{array}$ \\
\hline
\end{tabular}




\begin{tabular}{|c|c|c|c|}
\hline respuesta & $\begin{array}{l}\text { 15. áreas de pasturas naturales } \\
\text { 16. áreas de cobertura vegetal } \\
\text { 17. área con alta biodversidad o con } \\
\text { especies endemicas } \\
\text { 18. área de degradcion territorial } \\
\text { 19. suelo cultivable } \\
\text { 20. suelo cultivable de alta calidad } \\
\text { 21. ecosistema diversidad } \\
\text { 22. consumo de agua } \\
\text { 23. inversión en el área protegida } \\
\text { 24. área de suelos alta calidad protegido } \\
\text { 25. restauración de suelos degradados } \\
\text { 26. inversión en reciclado de suelos y agua }\end{array}$ & $\begin{array}{l}\% \\
\% \\
\% \\
\% \\
\% \\
\mathrm{n}^{\circ} / \mathrm{km}^{2} \\
\mathrm{~m}^{3} / \mathrm{persona}_{\mathrm{an} o} \\
\mathrm{pesos} / \mathrm{m}^{2} / \mathrm{año} \\
\% \\
\% \\
\text { pesos/m²/año }\end{array}$ & $\begin{array}{l}a-c \\
a-c \\
a-b-c \\
b-c-d \\
a-c \\
a-c \\
a-b-c \\
c-d \\
d \\
c-d \\
d \\
c-d\end{array}$ \\
\hline \begin{tabular}{|} 
sumidero de residuos \\
presión \\
estado \\
\\
respuesta
\end{tabular} & $\begin{array}{l}\text { 27. residuos urbanos } \\
\text { 28. potencial contaminación industrial } \\
\text { 29. consumo anual de agua } \\
\text { 30. uso agrícola campos } \\
\text { 31. cantidad de ganado } \\
\text { 32. área c residuo de actividad minera } \\
\text { 33. área industrial } \\
\text { 34. metales pesados en el agua } \\
\text { 35. nitratos en fuente de agua potable } \\
\text { 36. cant de personas tratadas por dif } \\
\text { respiratorias } \\
\text { 37. tratamiento de aguas recicladas } \\
\text { 38. acción para mitigar contam } \\
\text { 39. cultivos orgánicos } \\
\text { 40. aire mitigación }\end{array}$ & $\begin{array}{l}\mathrm{tn} / \mathrm{km}^{2} / \mathrm{años} \\
\mathrm{n}^{\circ} / \mathrm{km}^{2} \\
\mathrm{n}^{\circ} / \mathrm{km}^{2} \\
\mathrm{n}^{\circ} / \mathrm{km}^{2} \\
\mathrm{n}^{\circ} / \mathrm{km}^{2} / \mathrm{año} \\
\% \\
\mathrm{ppm} \\
\text { cant } / \mathrm{km}^{2} \\
\% \\
\mathrm{n} \text { actividades } / \mathrm{km}^{2} / \text { year } \\
\% \\
\%\end{array}$ & $\begin{array}{l}d \\
b-d \\
d \\
a-d \\
d \\
d \\
b-d \\
c-d \\
c-d \\
c-d \\
c-d \\
b-c-d \\
c-d \\
c-d\end{array}$ \\
\hline \begin{tabular}{|} 
actividades y servicios \\
(construcciones) \\
presión \\
estado \\
\\
respuesta \\
\end{tabular} & $\begin{array}{l}\text { 41. densidad de población } \\
\text { 42. edificaciones en zonas de riesgo } \\
\text { 43. rutas y caminos en zona de riesgo } \\
\text { 44. suelos sin limitaciones } \\
\text { 45. áreas naturales en riesgo } \\
\text { 46. áreas en zona de riesgo }\end{array}$ & $\begin{array}{l}\mathrm{hab} / \mathrm{km}^{2} \\
\% \\
\% \\
\% \\
\%\end{array}$ & $\begin{array}{l}\text { d } \\
\text { b-d } \\
\text { a-d } \\
\text { a-d } \\
\text { b-c-d } \\
\text { a-b-d }\end{array}$ \\
\hline \begin{tabular}{|c|} 
actividades y servicios \\
(l turismo) \\
presión \\
estado
\end{tabular} & $\begin{array}{l}\text { 47. número de visitantes } \\
\text { 48. áreas de playas } \\
\text { 49. sitios de interés cultural } \\
\text { 50. atractivos turísticos } \\
\text { 51. territorios degradados } \\
\text { 52. zonas de parques públicos } \\
\text { 53. inversión mantenimiento de áreas } \\
\text { naturales }\end{array}$ & $\begin{array}{l}\mathrm{n} / \mathrm{km}^{2} / \mathrm{año} \\
\mathrm{m}^{2} / \mathrm{km}^{2} \\
\mathrm{n} / 10 \mathrm{~km}^{2} \\
\mathrm{n} / 10 \mathrm{~km}^{2} \\
\% \\
\% \\
\% / \mathrm{km}^{2} / \mathrm{año}\end{array}$ & $\begin{array}{l}\text { d } \\
\text { a-c } \\
\text { d } \\
\text { a-d } \\
\text { b } \\
b \\
\text { d }\end{array}$ \\
\hline
\end{tabular}


La tercera etapa consiste en el cálculo de los índices agregados de presión, estado y respuesta (a su vez se incluyen indicadores de fuerzas motrices e impactos dentro de estos) para cada una de las cuatro funciones mencionadas de acuerdo a:

$$
\mathrm{INp}=\Sigma \mathrm{Vi} \cdot \mathrm{Wi}
$$

Donde INp es el índice de presión sobre la condición natural del ambiente; Vi es el valor normalizados del indicador y $\mathrm{Wi}$ es el peso del indicador; $\mathrm{i}=(1-\mathrm{n}), \mathrm{n}$ es el número total de indicadores presión.

De forma similar se procede para el cálculo de índices de estado y de respuesta, tanto para naturalidad como para las funciones fuente, sumidero, actividades-servicios.

Los índices de presión, estado y respuesta se integran en índices de cada una de las funciones:

$$
\mathrm{IN}=(\mathrm{INp}+\mathrm{INs}+\mathrm{INr}) / 3
$$

Donde IN el indice de la función naturalidad; INp el índice de presión sobre la naturalidad, INe el índice de estado de la naturalidad y INr el índice de respuesta sobre la naturalidad.

Procediéndose igualmente para cada una de las funciones abordadas: IR: índice de la función fuente de recursos, IW: índice de la función sumidero de residuos, ISS: índice de la función actividades - servicio.

Finalmente se calcula el índice de calidad ambiental (EQI) obtenido a partir de la siguiente expresión

$$
\mathrm{EQI}=(\mathrm{IN}+\mathrm{IR}+\mathrm{IW}+\mathrm{ISS}) / 4
$$

Donde IN es el índice de naturalidad; IR Índice de fuente de recursos; IW Índice de sumidero de residuos y ISS el Índice de actividades/servicios.

La interpretación del valor final del índice de calidad ambiental, está expresado en una escala de cinco clases cuyo valor máximo es uno y el mínimo cero, correspondiendo los valores más altos a la situación ambiental más optima (Tabla 2).

Tabla 2

CATEGORÍAS DEL ÍNDICE DE CALIDAD AMBIENTAL

\begin{tabular}{|c|c|c|}
\hline Clases & Valor & Calidad \\
\hline Clase 1 & $0.01-0.19$ & baja \\
Clase 2 & $0.20-0.39$ & moderadamente baja \\
Clase 3 & $0.40-0.59$ & media \\
Clase 4 & $0.60-0.79$ & moderadamente alta \\
Clase 5 & $0.80-1.00$ & alta \\
\hline
\end{tabular}

Para poder comparar resultados desde el punto de vista metodológico se elaboraron los índices a partir de diferentes niveles de análisis. Se establecieron listados acotados e integrados de indicadores para todas las dimensiones incluidas (Tabla 3). 
Tabla 3

LISTADO SIMPLIFICADO DE INDICADORES

\begin{tabular}{|c|c|c|}
\hline Variables & indicadores & unidades \\
\hline $\begin{array}{l}\text { naturalidad } \\
\text { presión } \\
\text { estado } \\
\text { respuesta }\end{array}$ & $\begin{array}{l}\text { 1. densidad de población } \\
\text { 2. cobertura vegetal natural } \\
\text { 3. área protegida }\end{array}$ & $\begin{array}{c}\mathrm{hab} / \mathrm{km}^{2} \\
\% \\
\%\end{array}$ \\
\hline $\begin{array}{c}\text { fuentes de recursos } \\
\text { presión } \\
\text { estado } \\
\text { respuesta }\end{array}$ & $\begin{array}{l}\text { 4. suelos cultivados } \\
\text { 5. agricultura } \\
\text { 6. protección suelos }\end{array}$ & $\begin{array}{l}\% \\
\% \\
\%\end{array}$ \\
\hline $\begin{array}{c}\text { sumidero de residuos } \\
\text { presión } \\
\text { estado } \\
\text { respuesta } \\
\end{array}$ & $\begin{array}{l}\text { 7. producción de residuos urbanos } \\
\text { 8. basurales, depósitos } \\
\text { 9. tratamiento de residuos }\end{array}$ & $\begin{array}{c}\mathrm{tn} / \mathrm{km}^{2} \\
\% \\
\text { monto }\end{array}$ \\
\hline $\begin{array}{c}\text { actividades y servicios }(\mathbf{C}) \\
\text { presión } \\
\text { estado } \\
\text { respuestas }\end{array}$ & $\begin{array}{l}\text { 10. edificaciones en áreas vuln } \\
\text { 11. área de construcciones } \\
\text { 12. planificación en áreas protegidas }\end{array}$ & $\begin{array}{l}\% \\
\% \\
\%\end{array}$ \\
\hline
\end{tabular}

\section{Resultados y discusión}

Los diferentes índices obtenidos a partir de los indicadores en el listado completo presentan diferencias significativas entre las localidades de San Antonio Oeste (SAO), San Antonio Este (SAE) y Las Grutas (LG). Cabe destacar que cada una de ellas forma parte de la misma unidad administrativa municipal aunque el Balneario Las Grutas y San Antonio Este poseen sendas delegaciones. Se enuncia esta característica dado que los indicadores de respuesta corresponden a las acciones gubernamentales con similar criterio para las tres localidades.

\subsection{Análisis del Índice de Naturalidad}

El índice presenta un valor de 0.40 para San Antonio Oeste, mientras que el valor de Las Grutas es el mínimo obtenido con 0.38. Para San Antonio Este el valor es de 0.33. Para componer estos resultados las dimensiones de estado y respuesta prevalecieron en San Antonio Oeste con valores que oscilaron entre 0.43 y 0.39 . Estos valores corresponden al grado de intervención antrópica en las geoformas del área y en la función y ejecución de las pautas de manejo a través del Plan de Manejo que regula esta localidad (Figura 2). Los valores del índice refieren a la perdida de naturalidad de los ecosistemas involucrados. La contaminación de las aguas y sedimentos es advertida desde 1994, los factores que contribuyen a la misma son principalmente los metales pesados que provienen de la pila de escoria localizad en cercanías de SAO. Estos elementos se acumulan en los moluscos que luego son consumidos por la población (Vázquez et al., 2007).

Para el sector de San Antonio Este los valores de esas dimensiones oscilaron entre 0.39 y 0.36 respectivamente, donde se aprecia también que en los alrededores de ese lugar los 


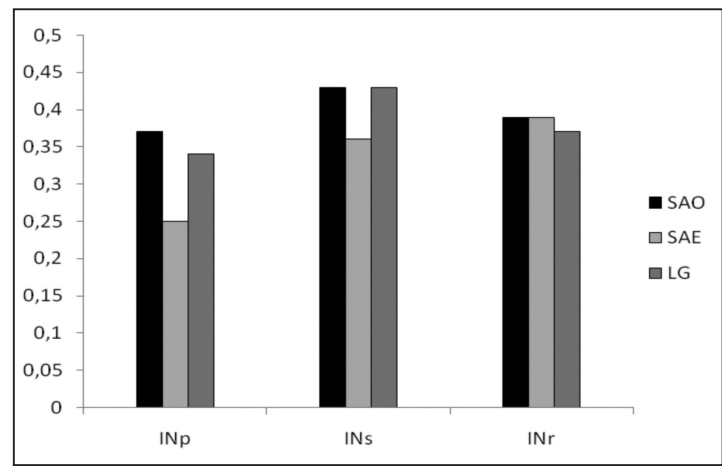

FIGURA 2. Índice de Naturalidad para las tres localidades analizadas.

ambientes naturales no mantienen su condición. En el Balneario Las Grutas el índice de naturalidad tiene el valor más bajo. Las dimensiones que lo conformaron oscilaron entre 0.34 y 0.42 para los indicadores de presión y respuesta respectivamente. En las tres localidades el valor más alto del índice corresponde al estado de los ecosistemas involucrados en sendos ambientes, corresponde $0.43,0.36$ y 0.37 para san Antonio Oeste, San Antonio Este y las Grutas respectivamente. Los valores más bajos del índice de naturalidad en cuanto a la dimensión estado reflejan la pérdida de la condición natural de estos ambientes, asociado principalmente a la presión que ejerce el incremento demográfico. La tasa de crecimiento desde el 2001 a la actualidad se estima en un 24\% para SAO y más del $46 \%$ en SAE (Municipalidad de San Antonio, 2008). Los valores de respuesta en este índice están estrechamente relacionado con las medidas de fiscalización y control en actividades extractivas tanto pesqueras como mineras (Ley 2669/94).

También se analizo el índice de Naturalidad para el sector noreste de San Antonio Oeste, el sector sur de SAE y las playas del sur de LG. Los resultados arrojados mejoraron la situación en cuanto al estado de estos ambientes. Para la primera localidad el índice es de 0,68, para SAE 0,65 y para LG ronda 0,62. Estos valores marcan la condición de naturalidad moderadamente alta, correspondiendo a lugares que no poseen presión demográfica y están presentes en la agenda de conservación.

\subsection{Indice Fuente de Recursos}

El índice que involucra al medio, como fuente de recursos IR presenta el valor más alto para la localidad de San Antonio Oeste, donde se concentra la mayoría de las actividades económicas productivas del sector con un valor de 0.49 (Figura 3). Representando para este índice el dato más bajo el de los indicadores de presión con 0.42 correspondiente con la diversidad de usos del suelo de esta localidad y área adyacente (ganadería, olivares, agricultura, extracción de áridos, marisquería, pesca). Las pesquerías artesanales en SAO se sustentan en una gran variedad de invertebrados (almeja púrpura vieira tehuelche, mejillón, cholga y pulpito patagónico) y peces (pejerreyes, róbalo, sargo, mero salmón de mar). Es de pequeña escala, tanto por los volúmenes capturados como por los beneficios económicos generados. Sin embargo, analizadas globalmente, las pesquerías artesanales involucran a más de 200 pescadores, entre recolectores costeros, pescadores de rastra y buzos (Narvarte et al., 2006). Uno de los objetivos más importantes en el plan de manejo es compatibilizar el concepto de extracción de los recursos mencionados con la conservación y sustentabilidad. 


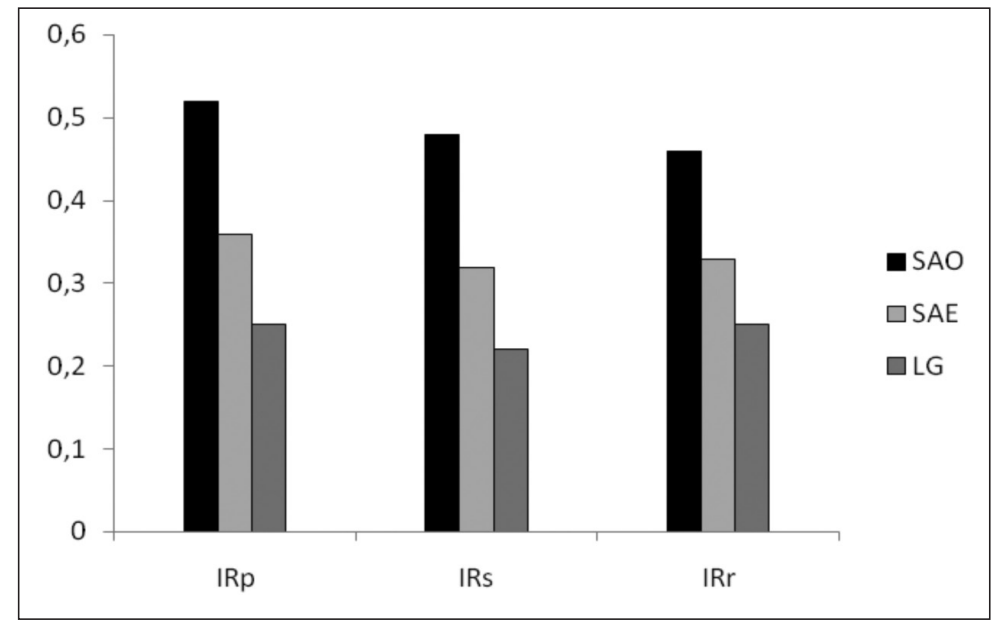

FIGURA 3. Índice Fuente de Recurso para las tres localidades analizadas.

Los indicadores de estado y respuesta como se observa en la figura 3 oscilan entre 0.48 y 0.46 debido al aumento en la importancia en la gestión de conservación de esta área, esta condición también se advierte en la nueva legislación vigente (Res 354/07) que regula las actividades extractivas, turísticas, de investigación y de expansión urbana entre otras. Los valores de IR para San Antonio Este y las Grutas oscila entre 0.34 y 0.24 respectivamente. Las dimensiones que alcanzaron valores mayores para estas localidades corresponden a los indicadores de presión y respuesta con valores que oscilan entre 0.36 y 0.25 ).

El análisis de este índice para los sectores aledaños a las urbanizaciones también revelaron números más altos con respecto al estado de los mismos, arrojando valores de 0,72, 0,64 y 060 para SAO, SAE y LG respectivamente. Se presentan estos lugares como potenciales recursos turísticos y zona de importancia de biodiversidad (aves, peces, mamíferos marinos, invertebrados, etc).

\section{3. Índice Sumidero de Residuos}

Con respecto al índice que considera al medio como sumidero de residuos IW el máximo valor corresponde también a San Antonio con 0.54. Este dato coincide con la presión que ejerce sobre el medio una población de más de 16.000 habitantes (INDEC, 2001), donde existe proliferación de basurales clandestinos en los terrenos aledaños a un canal de marea típico de esta localidad y donde se asienta una pila de escoria desde hace más de tres décadas con abundancia de plomo, zinc, cadmio, etc. El material residual de actividades mineras está sometido continuamente a distintos procesos: infiltración a capas subterráneas y lixiviación hacia el canal de marea cuando precipita (Figura 4). Esta condición se refleja en el índice de estado de esta parte de los ambientes en SAO, correspondiendo a un valor más bajo dentro de todas las dimensiones $(0,27)$ acercándose a la peor situación observada.

Las dimensiones que conformaron este índice arrojaron valores más altos las correspondientes a presión y respuesta alcanzando valores de 0.52 y 0.57 para la segunda variable mencionada. Cabe destacar que en esta localidad se ha completado el tendido de la red de 


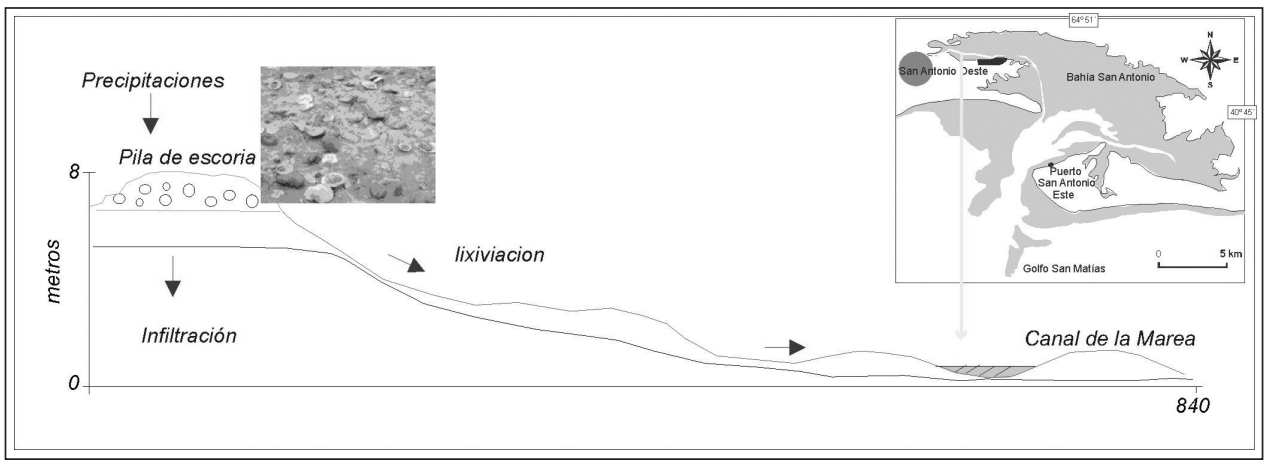

FiguRa 4. Perfil transversal del sector de escorias en la localidad de San Antonio Oeste.

saneamiento (año 2008) y se han tomado medidas muy severas con respecto a las fábricas de harina de pescado que funcionan en la planta industrial de dicha localidad (Municipalidad de San Antonio, 2009). Además también se invertirá en la construcción de una planta de tratamiento de residuos sólidos urbanos como parte del saneamiento de residuos (Municipalidad de SAO, 2008). Para Las Grutas el valor es de 0.31 y para San Antonio Este alcanza el mínimo valor con 0.24. Las variables que sobresalen principalmente en este índice corresponden a la presión y respuesta, con valores que van desde 0.35 a 0.28 para la primera localidad y desde 0.29 a 0.20 para SAE (Figura 5). La variable de estado para SAO y LG se mantiene en un rango de valores más bajo lo que representa acercarse a una situación no tan óptima de acuerdo a la escala considerada. Con respecto a los lugares adyacentes a las localidades analizadas al no existir asentamientos humanos no se evaluó esta categoría. In situ se observó que a partir del 2006 las tareas de limpiezas y mantenimiento de espacios circundante a estas playas se realiza constantemente.

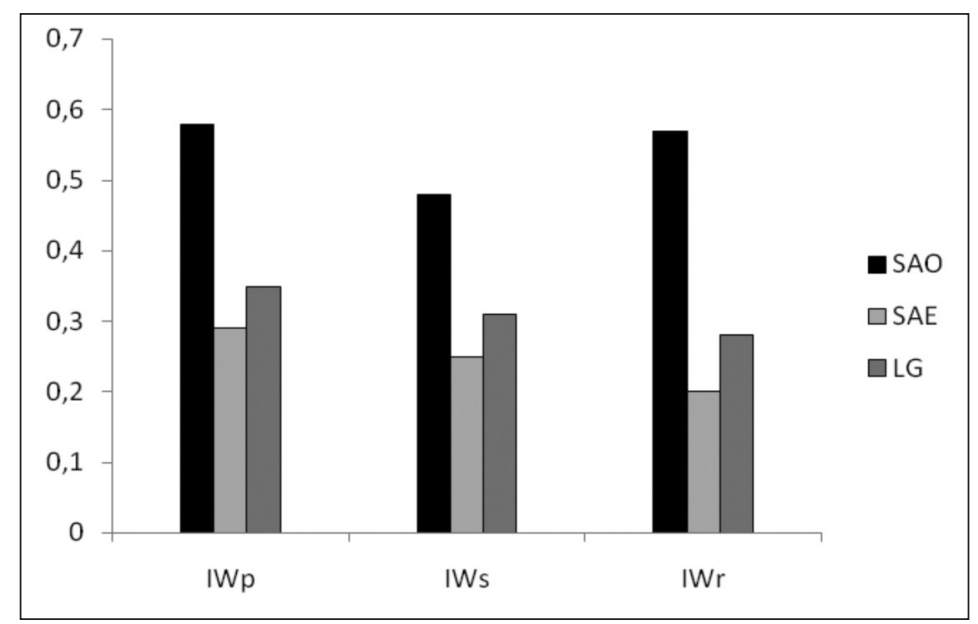

FIGURA 5. Índice Sumidero de Residuos para las tres localidades analizadas. 


\section{4. Índice de Soporte de actividades y servicios}

La capacidad del medio como soporte de actividades y servicios a través del ISS presentó una variabilidad importante de valores principalmente entre San Antonio Oeste y Las Grutas. En este caso se consideraron las actividades y servicios derivadas de la construcción (Ac) y el turismo (T). Para la primera variable considerada se observan los índices más óptimos para SAO con valores que oscilan entre 0.41 y 0.54 , mientras que para el balneario las Grutas el índice presenta valores mucho más bajos que oscilan entre 0,20 y 0,41 (Figura 6a). Cabe destacar que en dicha localidad la mayor parte de las construcciones se halla sobre la costa acantilada, lo que ejerce un mayor impacto sobre estas geoformas. El plano catastral del balneario Las Grutas presenta dos patrones de distribución sobre la costa: un primer sector localizado en el noreste donde se observa una ocupación espacial espontanea caótica y concentrada, un segundo sector hacia sudoeste donde se observan normas de ordenamiento territorial planificado medido respetando la línea de edificación retirada de la zona costera vulnerable (Figura 6).

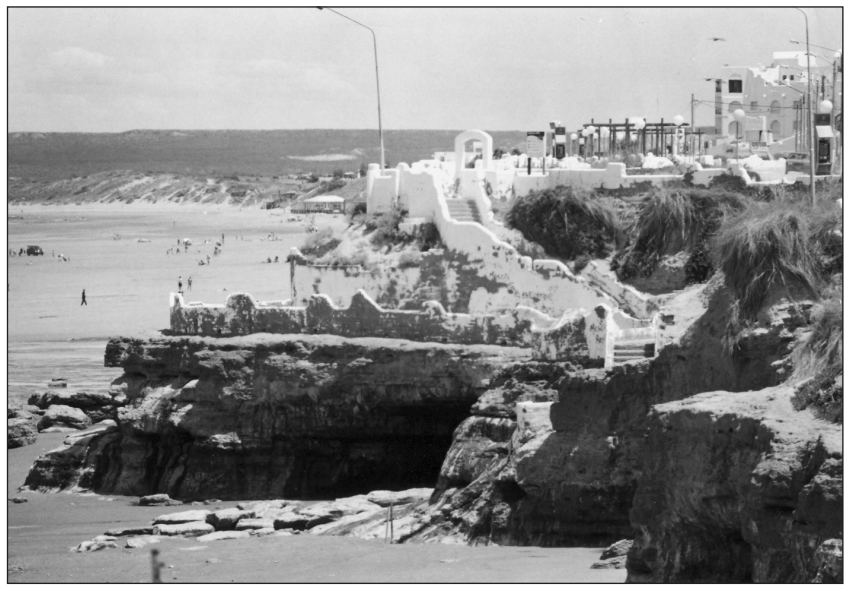

FIGURA 6: Edificaciones construidas sobre la costa acantilada de Las Grutas.

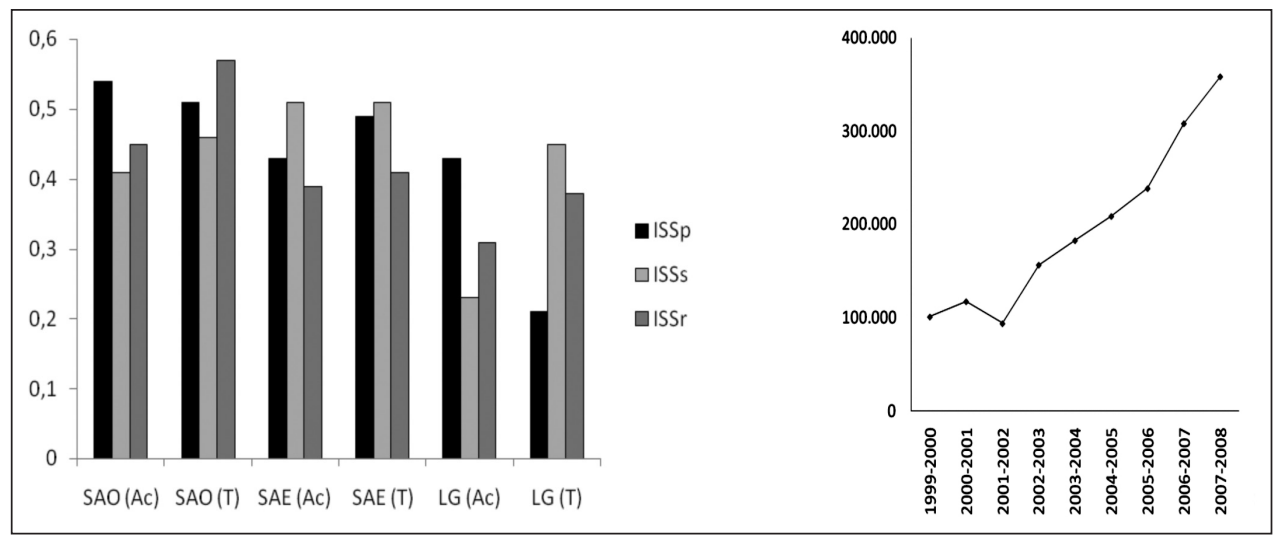

FIGURA 7. a Índice de Actividades y Servicios para los rubros de construcción y turismo comparados. b. Evolución de la cantidad de turistas en el área de bahía San Antonio (Secretaria de Turismo, 2008). 
La segunda variable analizada está relacionada con la actividad turística desarrollada en estas localidades. El balneario las Grutas presenta el índice más bajo para esta actividad con un valor de 0,37, mientras que para San Antonio Oeste y San Antonio Este varían entre 0,47 y 0,51 . Estos resultados están relacionados con una afluencia masiva de visitantes durante los periodos estivales. El incremento de la actividad turística es de un $32 \%$ como se observa en la figura 6b. El número de visitantes para el año 2008 supero los 350.000 (Municipalidad de San Antonio Oeste, 2008).

Los índices agregados para estas áreas también se obtuvieron a partir de listados simplificados de indicadores. Los resultados de la aplicación de 12 indicadores previamente seleccionados, presentó valores mayores con respectos a los analizados anteriormente. El índice de naturalidad para San Antonio Oeste resultó de 0,62. Prevaleciendo la dimensión correspondiente a estado y respuesta. Para San Antonio Oeste fue de 0,35 y de 0,24 para Las Grutas. Los índices que involucran al medio como fuente de recursos, sumideros de residuos y como soporte de diferentes actividades y servicios variaron ampliamente entre las tres localidades como se observa en la tabla 4.

Tabla 4

LISTADO SIMPLIFICADO DE INDICADORES

\begin{tabular}{|c|c|c|c|c|c|c|c|}
\hline Variables & Indicadores & Unidad & $\min$ & $\max$ & SAO & SAE & LG \\
\hline naturalidad & & & & & \multirow{4}{*}{0,62} & \multirow{4}{*}{0,35} & \multirow{4}{*}{0,24} \\
\hline presión & $\begin{array}{l}\text { 1. densidad de rutas y } \\
\text { caminos }\end{array}$ & $\mathrm{km} / \mathrm{km}^{2}$ & 0 & 5 & & & \\
\hline estado & $\begin{array}{l}\text { 2. cambios en la } \\
\text { cobertura vegetal }\end{array}$ & $\%$ & 0 & 100 & & & \\
\hline respuesta & 3. área protegida & $\%$ & 1 & 100 & & & \\
\hline \multicolumn{8}{|l|}{ fuentes de recursos } \\
\hline presión & $\begin{array}{l}\text { 4. población en suelos } \\
\text { aptos }\end{array}$ & $\mathrm{n} / \mathrm{ha}$ & 1 & 12 & \multirow{3}{*}{0,71} & \multirow{3}{*}{0,42} & \multirow{3}{*}{0,37} \\
\hline estado & 5. área productiva & $\%$ & 0 & 23 & & & \\
\hline respuesta & $\begin{array}{c}\text { 6. inversión en área } \\
\text { protegida }\end{array}$ & monto anual & 10.000 & 600.000 & & & \\
\hline \multicolumn{8}{|l|}{$\begin{array}{l}\text { sumidero de } \\
\text { residuos }\end{array}$} \\
\hline presión & 7. áreas de depósito & $\%$ & 1 & 9 & \multirow{3}{*}{$\mathbf{0 , 5 8}$} & \multirow{3}{*}{0,36} & \multirow{3}{*}{$\mathbf{0 , 3 3}$} \\
\hline estado & 8. residuos urbanos & tn/años & 200 & 900000 & & & \\
\hline respuesta & $\begin{array}{l}\text { 9. inversión en } \\
\text { saneamiento }\end{array}$ & monto/anual & 130000 & 1200000 & & & \\
\hline \multicolumn{8}{|l|}{$\begin{array}{l}\text { actividades y } \\
\text { servicios }\end{array}$} \\
\hline presión & $\begin{array}{l}\text { 10. densidad de } \\
\text { población }\end{array}$ & $\mathrm{hab} / \mathrm{km}^{2}$ & 1 & 1,7 & \multirow{3}{*}{0,56} & \multirow{3}{*}{0,47} & \multirow{3}{*}{0,39} \\
\hline estado & 11. áreas con riesgos & $\%$ & 0 & 6 & & & \\
\hline respuestas & $\begin{array}{l}\text { 12. inversión en } \\
\text { mitigación }\end{array}$ & monto/anual & 10000 & 450000 & & & \\
\hline
\end{tabular}

Asimismo variaron significativamente los valores de las dimensiones que constituyen estos índices en las tres localidades prevaleciendo en la mayoría la presión y el estado sobre la respuesta involucrada. Esta condición se presenta debido a que el peso que se otorga a los indicadores de respuesta como a las otras dimensiones es 1 y considerando que estos 
sitios dependen de la misma unidad administrativa municipal las decisiones políticas y las inversiones públicas son similares para las tres localidades. Los resultados obtenidos con la lista acotada de indicadores son visiblemente mayores que los obtenidos con el listado desagregado de indicadores.

\section{5. Índice de Calidad Ambiental}

La obtención de los índices desagregados permitió zonificar el área de estudio de acuerdo a los valores del EQI en cuatro clases. Cada una de estas pertenece a una zona específica de la reserva natural Bahía San Antonio. El valor más alto corresponde a San Antonio Oeste con 0.46, le sigue San Antonio Este con un valor de 0.34 y Las Grutas posee un índice de 0.28 (Figura 8). Estos resultados surgen de la interrelación de todos los índices analizados anteriormente empleando el listado completo de indicadores donde se calcularon todas las dimensiones del modelo DPSIR. Los valores obtenidos permitieron zonificar el área de la siguiente manera: La localidad de San Antonio Oeste corresponde a la clase 3 que representa una calidad ambiental media, donde se refleja una mayor actividad humana relacionada con el aumento de la población. La presión ejercida sobre el medio físico a partir de la extracción de recursos mineros y pesqueros, el vertido de efluentes cloacales sin tratamientos, la localización de escorias en el suburbio de SAO principalmente altera la condición natural de los ambientes. El uso de los indicadores de respuesta mostró valores altos que ponen en evidencia la actual preocupación para comenzar a contrarrestar las presiones sobre del medio.

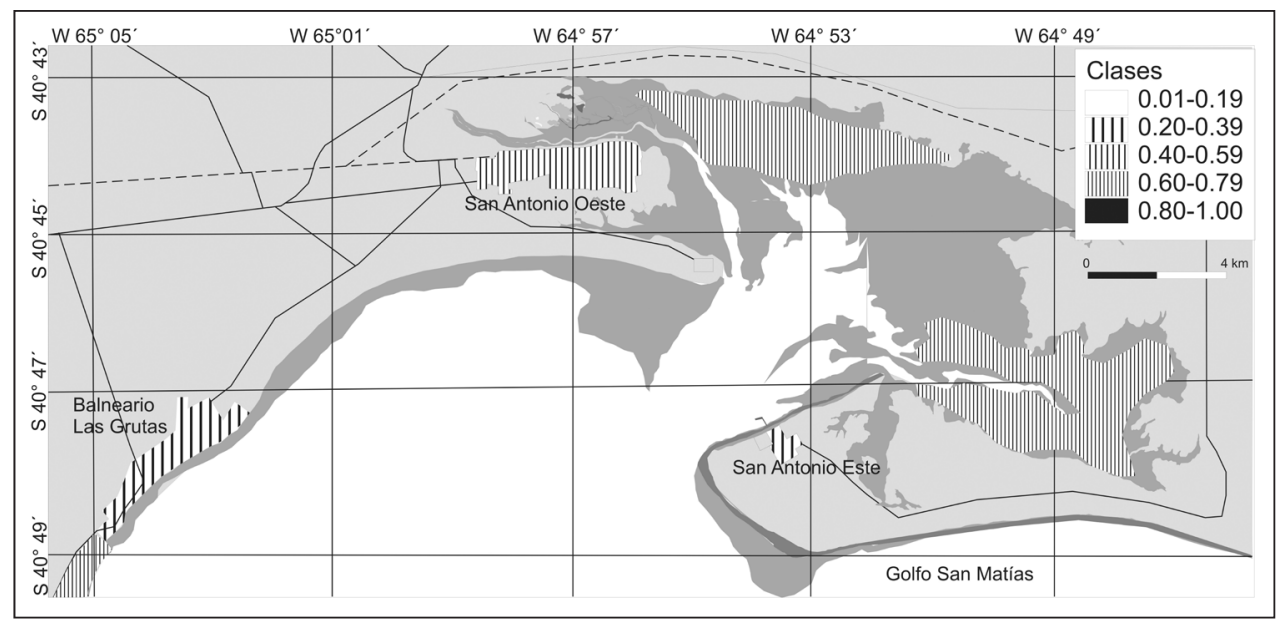

FIGURA 8. Zonificación de acuerdo al Índice de Calidad Ambiental (EQI).

La zona de San Antonio Este posee una calidad dentro de la clase 2, calificada como moderadamente baja. En esta zona portuaria donde la población creció en un $60 \%$ desde 2001, las actividades económicas aumentaron, los índices de presión reflejaron el impacto de las mismas sobre los diferentes ambiente. No obstante los índices de respuestas no fueron muy altos. Si bien corresponde a la misma administración pública no existe un marcado accionar para la mitigación en este lugar. Aunque los índices de estado marcaron una mejor condición de naturalidad con respecto a San Antonio Oeste. 
El Balneario Las Grutas posee valores más bajos de calidad ambiental, lo encuadran dentro de la categoría baja. Los índices obtenidos en este lugar muestran una gran actividad turística anual, una planificación urbana diferenciada y sectores con problemas de erosión marcados principalmente en los acantilados. Los indicadores de respuestas muestran un incipiente esfuerzo por mitigar los impactos principalmente en la zona costera.

Los sectores adyacentes a las urbanizaciones analizadas presentaron los mejores valores del índice de calidad ambiental ubicándolos en la clase 4 moderadamente alta. El sector noreste de SAO se caracteriza por unidades geomorfológicas diversas (planicies de mareas, marismas canales de mareas secundarios, salitrales, etc) por una escasa actividad y una accesibilidad dificultosa. La circulación del flujo de agua, con corrientes de reflujo dominantes renueva continuamente el medio acuático (Isla et al., 1995, Schnak et al, 1998 Carbone et al., 2008, Moreira et al., 2009). El sector aledaño a SAE en el este de la bahía también se caracteriza por la escasa concentración de actividades, aunque en varios sectores cercanos a Caleta Falsa se realizaba extracción de áridos en canteras (actualmente controlada), también posee una diversidad importante de geoformas como playas, planicies y canales, marismas. El sector de las playas al sur de LG posee condiciones más óptimas en cuanto a la condición de calidad ambiental.

Asimismo en cada una de las zonas determinadas se puede diferenciar puntos conflictivos donde se deberían implementar acciones directas para restaurar, mitigar, conservar y rehabilitar sectores vulnerables a las presiones ejercidas (Figura 9). En la zona de San Antonio Oeste un área a remediar urgente es la zona de los depósitos de escorias en el suburbio de la ciudad (1). Otra área a considerar para rehabilitar es la franja circundante al canal La Marea donde se localizan los basurales clandestinos (2).

La zona a conservar del impacto humano es la vasta planicie de marea y marismas del sector Este de la ciudad, con sus canales de marea (3). Otro sector a considerar es el del puerto San Antonio Este donde se debe controlar la actividad portuaria y pesquera (4). La zona aledaña correspondería a conservación neta sin uso (3). En el sector del balneario las Grutas las acciones de manejo serían: sector céntrico restricción total de edificaciones sobre la costa acantilada, remediación de bajadas a la playa con infraestructura débil (5) y una zona de conservación es la correspondiente a las playas del norte y del sur (3). Las

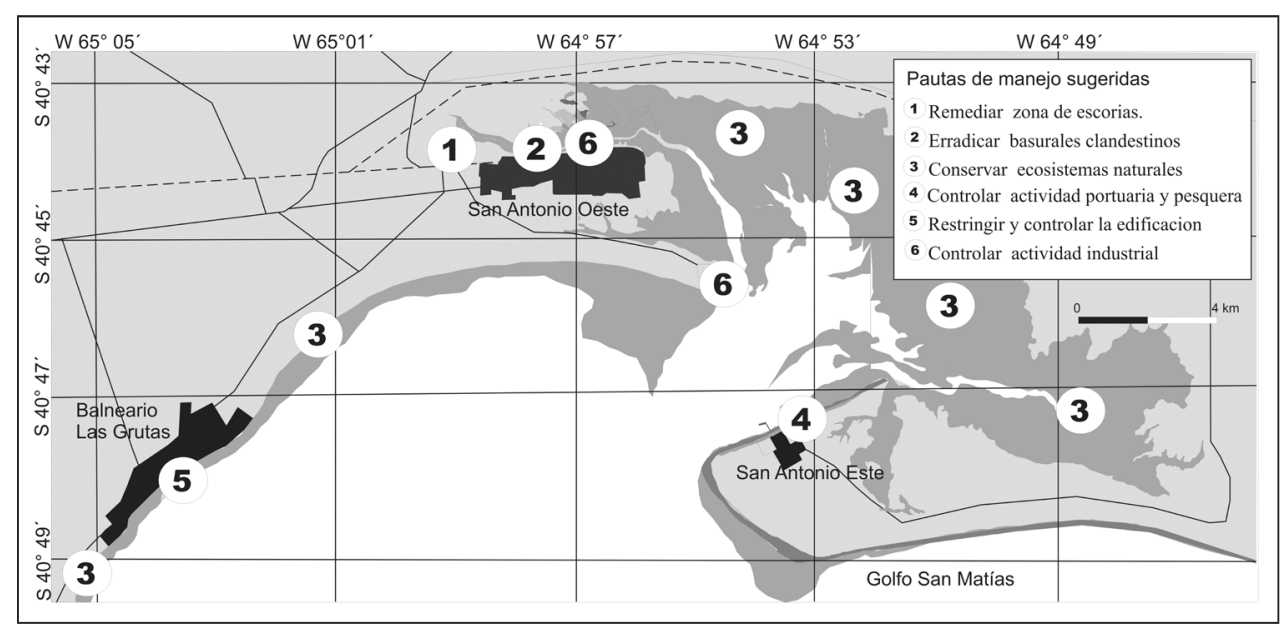

FIGURA 9. Cartografía de las pautas de manejo sugeridas para la reserva San Antonio. 
pautas de manejo integrado que se deben aplicar en esta zona costera están relacionadas a: la conservación de los ecosistemas; rehabilitación de zonas utilizadas, usos y apropiación de los recursos naturales renovables con técnicas de bajo impacto con una administración eficiente y sustentable de los mismos.

\section{Conclusiones}

Los índices obtenidos a través de los indicadores posibilitaron la organización, sistematización, cuantificación y comunicación de la información relativa de distintos aspectos propios de esta zona costera lo cual resulta básico para la toma de decisiones en relación a políticas ambientales a aplicar en un futuro cercano. La variabilidad de sitio, infraestructura, equipamiento, procesos naturales y antrópico actuantes en cada una de las unidades consideradas permitió zonificar el área de acuerdo a los valores del índice ambiental obtenidos.

La aplicación del Índice de Calidad Ambiental determinó que la reserva natural Bahía San Antonio posee una calidad ambiental de media a moderadamente baja de acuerdo a los resultados obtenido a partir de los índices agregados (IN,IR,IW,ISS). La localidad de San Antonio posee el valor más alto $(0,46)$ mientras que San Antonio Este y las Grutas alcanzaron los valores más bajos, entre 0,36 y 0,29. La calidad ambiental mas optima la presentaron las zonas aledañas a las urbanizaciones consideradas, donde la presión demográfica y las actividades productivas son escasas.

La interrelación de los indicadores DPSIR y las diversas funciones del medio permitió la zonificación obtenida a partir del EQI donde se determinó cuáles son los sectores en esta área que necesitan la implementación rápida de pautas estratégicas de manejo. Los índices obtenidos indicaron falta de condición natural de estos ecosistemas por presión antropica, generando una sobreexplotación del medio físico, contaminación del mismo y a su vez se pudo inferir la escasa aplicación de políticas ambientales y urbanas en el pasado cercano. De acuerdo al índice de respuesta, algunos indicadores marcan un cambio en cuanto a la formulación y aplicación de medidas sustentables para poder modificar las situaciones presentes y pasadas.

El monitoreo continuo de la condición ambiental de las zonas costeras es fundamental para orientar las acciones y políticas a implementar. En la reserva Bahía San Antonio la aplicación del EQI permitió observar un área vulnerable y en riesgo ambiental, pero también surge de este estudio que hay un incipiente cambio en política de conservación y adecuado manejo de recursos. Este tipo de estudios se debe realizar continuamente debido a la dinámica de este ambiente costero. La aplicación de políticas ambientales apropiadas se verá reflejada quizás en la siguiente evaluación de la calidad ambiental de esta zona patagónica. Los resultados obtenidos indican que no es tarde para revertir la situación ambiental de esta área costera.

\section{Referencias bibliograficas}

BAAN P. J. AND VAN BUUREN J. T. (2003) Testing of indicators for the marine and coastal environment in Europe. Technical report European Environmental Agency Part 3,1-65.

BECKER, A. (2006). Geomorphic consequences of urban development and mining activities an analysis of study areas in Spain and Argentina. Geomorphology, 73, 3-4: 185-206.

BERGER, A.R. AND LIVERMAN, D.G. (2002). Geoindicators for ecosystem monitoring in parks and protected areas. Parks Canada, Ecosystem 404 Science review reports 18, 45 p.

BONACHEA, J.,V. M. BRUSCHI, REMONDO J., GONZALEZ A., SALAS L., BERTENS J., CENDRERO A., OTERO C. AND GIUSTI C., FABBRI A, GONZALEZ-LASTRAD 
J. R. AND ARAMBURU J. M. (2005) An approach for quantifying geomorphological impacts for EIA of transportation infrastructures: a case study in northern Spain. Geomorphology 66 95-117

CARBONE, M. E, PERILLO G. M. E. Y PICCOLO, M. C. (2007) Dinámica morfológica de los ambientes costeros de la bahía San Antonio, provincia de Río Negro. GEOACTA Vol 32 ISSN 0326-7237 pp. 59-73.

CARBONE M E, ALBERDI E., PERILLO G M E, PICCOLO M C AND DRAGANI W. C (2008) Circulation of the water flow in the main channel of San Antonio bay, argentina ECSA 44 Bahía Blanca 29 de setiembre 3 de octubre.

CENDRERO, A. AND D.W. FISCHER (1997). A procedure for assessing the environmental quality of coastal areas for planning and management. Journal of Coastal Research, 13, 3, 732-744.

CENDRERO, A. (2001). Proyecto ELANEM, un aporte a la elaboración de indicadores de calidad ambiental. V Congreso Latino-Americano de Ecología. Jujuy, Argentina. 21 pp

CENDRERO, A., DÍAZ DE TERAN, J.R., GONZALEZ, D., MASCITTI, V., ROTONDARO, R., AND TECCHI, R., (1993). Environmental diagnosis for planning and management in the high Andean region; the biosphere reserve of Pozuelos, Argentina. Environmental Management, 17 (5): 683-703.

CENDRERO, A., FRANCÉS, D. DEL CORRAL, J.L. FERMÁN, D. FISCHER,L. DEL RIO, M. CAMINO AND A. LÓPEZ, (2003) Indicators and indices of environmental quality for sustainability assessment in coastal areas; application to case studies in Europe and the Americas. Journal Coastal Reserch 19 (3): 919-933.

CHARLIER R.H. AND BOLOGA, A.S. (2003). Coastal zone under siege - is there realistic relief available? Journal of Coastal Research, 19(4), 884-889.

CHIRINO E., ABAD, J. AND BELLOT J. (2008). Uso de indicadores de Presión-EstadoRespuesta en el diagnóstico de la comarca de la Marina Baixa, SE, España Ecosistemas 17 (1): 107-114.

DADON, J. R. Y S. D. MATTEUCCI, (2007). Patrones de desarrollo costero en la Provincia de Buenos Aires. En: Crecimiento urbano y sus consecuencias sobre el entorno rural. El caso de la ecorregión pampeana. pp. 251- 279. GEPAMA, Ed. Orientación, Buenos Aires. ISBN 978-987-9260-45-6-

DADON, J. R., (2006). Diagnóstico de la zona costera argentina y perspectivas para su manejo. En: Conferencia Regional: Fortalecimiento de Capacidades para el Manejo Costero Integrado, MCI-Sur (eds.) Montevideo, Uruguay. Proyecto «Sustentabilidad de la Zona Costera Uruguaya» (AUCC-CIDA), Universidad de la República, Montevideo

DADON, J. R. Y S. D. MATTEUCCI, (2006). Caracterización de las grandes regiones costeras argentinas. En: Manual de Manejo Costero para la Provincia de Buenos Aires (F. I. Isla y C. Lasta, eds.), pp. 11-39. Editorial Universitaria de Mar del Plata, Universidad de Mar del Plata, Mar del Plata. ISBN 987-544-182-1-

EUROPEAN ENVIRONMENTAL AGENCY EEA (1998). Europe's environment: The second assessment. Copenhagen. $132 \mathrm{p}$.

GENCHI S A., CARBONE M. E, PERILLO G M E, PICCOLO M. C. (2008) Study of the temporary hydrographic networks as a conditioner of the urban morphology. Case Las Grutas beach resort ECSA 44 Bahía Blanca 29 de setiembre 3 de octubre.

INDEC. CENSO NACIONAL DE POBLACIÓN, HOGARES Y VIVIENDAS (2001). Cuadros 11.1 - 11.6 http://www.indec.gov.ar/censo2001s2_2/.

ISLA, F., E. SCHNACK Y S. ALIOTTA. (1995). Evolution and sedimentary dynamics of San Antonio Bay, Rio Negro, Argentina. XI Symposium on Coastal Sedimentology. Rio de Janeiro Anales 6 pp. 
JIMENEZ BELTRAN, D.,(2000). Los indicadores ambientales como instrumento de la política ambiental y para el desarrollo sostenible. In: LLANES, G.J. Estadística y medio ambiente, pp. 11-27. Instituto de Estadística de Andalucía, Sevilla.

LAREDO, I. M, AMERISO, C., CICARÉ, A., CIGNACCO, G., ROSA, I., ILLUNDAIN, L, JUDAIS, A. MUSSIO, V. (2001) Sistema de indicadores ambientales para el desarrollo sustentable del Mercosur Sextas Jornadas «Investigaciones en la Facultad» de Ciencias Económicas y Estadística Instituto de Investigaciones Teóricas y Aplicadas, Escuela de Contabilidad 401.

LIN T., XUE X., LU C. (2007). Analysis of Coastal Wetland Changes Using the «DPSIR» Model: A Case Study in Xiamen, China. Coastal Management, Volume 35, Issue 2, 289-303.

MAELFAIT, H.; BELPAEME, K.; LESCRAUWAET, A.K.; MEES, J. (2006). Indicators as reliable guides for Integrated Coastal Zone Management, in: Forkiewicz, M. 2006. Integrated Coastal Zone Management: theory and practice. EuroCoast - Littoral 180-186.

MARCOMINI S. C, LÓPEZ R A. Y SPINOGLIO A. (2007). Uso de la morfología costera como geoindicador de susceptibilidad a la erosion en costas cohesivas, Necochea, Buenos Aires Revista de la Asociación Geológica Argentina 62 (3): 396-404.

MOREIRA D., SIMIONATO C. G.,DRAGANI W. C. AND MARIO N. NUÑEZ (2009) Tidal and Residual Currents Observations at the San Matías and San José Gulfs, Northern Patagonia, Argentina Journal of Coastal Research 25(4): 957-968.

MUNICIPALIDAD DE SAN ANTONIO OESTE, (2008). Cantidad de turistas 2006-2007. Estadística cuantitativa. Obras públicas para la ciudad. http://www.sanantoniooeste. gov.ar/.

NARVARTE M.A., GONZÁLEZ R., FERNÁNDEZ M. (2006). Comparison of Tehuelche octopus (Octopus tehuelchus) abundance between an open-access fishing ground and a marine protected area: Evidence. Fisheries Research, 79: 112-119.

ORGANIZATION FOR ECONOMIC CO-OPERATION AND DEVELOPMENT (1993). OECD core set of indicators for environmental performance reviews. A synthesis report by the Group on the State of the Environment. Environment Monographs, $n^{\circ} 83$.

ORPÍ, A., JAIMEZ SALGADO J., OLIVERA ACOSTA E., VALDÉS HERNÁNDEZ J., GERTRUDIS DÍAZ, DE TERÁN J. Y SOTO TORRES, J. (2006) Sistema de indicadores para la evaluación de la calidad ambiental del Municipio Bauta, La Habana, Cuba. Universidad y Tecnología Volumen 10, No 41, 263-268.

PIRRONE, N. TROMBINO, G. CINNIRELLA, S. ALGIERI A. BENDORICCHIO G. AND PALMERI L., (2005). The Driver-Pressure-State-Impact-Response (DPSIR) approach for integrated catchment-coastal zone management: preliminary application to the Po catchment-Adriatic Sea coastal zone system Reg Environ Change 5: 111-137.

RIVAS, V.AND CENDRERO, A., (1991). Use of natural and artificial accretion in the north coast of Spain; historical trends and assessment of some environmental and economic consequences. Journal of Coastal Research. 7(2): 491-507.

RIVAS, V. AND CENDRERO, A., (1995). Human influence on a low-hazard coastal area; an approach to risk assessment and proposal of mitigation strategies. Journal of Coastal Research, Special issue No. 12: 289-298.

RIVAS V., REMONDO TEJERINA, J., SOTO TORRES, J., CENDRERO UCEDA, A. AND BONACHEA PICO, J. (2006). Sensitivity of landscape evolution and geomorphic processes to direct and indirect human influence» Geografia fisica y dinamica quaternaria, 29 pag. 125-137.

SCOPE (Scientific Committee on Problems of the Environment), (1995). Environmental indicators; a systematic approach to measuring and reporting on the environment in 
the context of sustainable development. In; Indicators of sustainable development for decision-making. Eds. N. Gouzee, B. Billharrz, Federal Planning Office, Bruselas: 1-25. SCHNACK, E.J., J.L. DEL RÍO Y F.I. ISLA. (1986). Formas y dinámicas del delta de reflujo de la bahía San Antonio, Río Negro. Actas de la primera reunión de sedimentología. La Plata 61-64.

SMN (Servicio Meteorológico Nacional). 2001. Estadísticas Meteorológicas 1991-2000. Buenos Aires.

UNDP (Commission on Sustainable Development), 1995. The role of indicators in decisionmaking. In; Indicators of sustainable development for decision-making, Eds. Gouzee, N., Mmazijn, B., and Billharz, S., Federal Planning Office, Brussels: 17 p.

VAZQUEZ N. N. GIL M. A.. ESTEVES J. L. AND NARVARTE M. A (2007) Monitoring Heavy Metal Pollution in San Antonio Bay, Rio Negro, Argentina Bull Environ Contam Toxicol 79: 121-125. 